\title{
EL LARGO CICLO DE LA REFORMA DE LA DIFERENCIACIÓN DE LA EDUCACIÓN SUPERIOR EN URUGUAY. DE LA UNIVERSIDAD SISTEMA AL SISTEMA UNIVERSITARIO (1984 - 2013)
}

\author{
Claudio Rama*
}

Recebido: 1 mar. 2014

Aprovado: 8 abr. 2014

*Universidad de la Empresa (UDE). Montevidéu, Uruguai. E-mail: claudiorama@gmail.com

Resumen: La educación superior en el Uruguay durante más de 150 años se redujo a una sola institución, la Universidad de la República, hasta 1984, cuando fue creada la primera universidad privada y la segunda universidad del país: la "Universidad Católica del Uruguay, Dámaso Antonio Larrañaga". A partir de ese momento, cambió la dinámica de la educación superior y se inició una "reforma de la diferenciación", que constituyó una lenta y polémica construcción de un sistema universitario desde una universidad - sistema. Fue un largo periodo de 29 años, concluido en el 2013, en el cual en un marco de tensiones y luchas políticas, se pasó desde un modelo universitario monopólico público, hacia un sistema universitario diferenciado público y privado. El presente ensayo, describe esos procesos; los articula a las dinámicas de América Latina; visualiza sus características propias; muestra la complejidad de las tensiones y luchas alrededor de la construcción de los sistemas universitarios y la define como una fase de la dinámica histórica universitaria del país.

Palabras claves: Diferenciación. Universidad. Universidades privadas. Universidades públicas. Reformas.

THE GREAT CYCLE OF DIFFERENTIATION REFORM OF HIGHER EDUCATION IN URUGUAY: FROM A ONE-UNIVERSITY SYSTEM TO A UNIVERSITY SYSTEM (1984-2013)

Abstract: Higher education in Uruguay for more than 150 years was reduced to a single institution, the University of the Republic, until in 1984 the first private university and the second university in the country was created. From that moment began a change in the dynamics of higher education, which we define as the reform of differentiation involving the slow and controversial construction of a university system. It was a period of 29 years, that ended in 2013, when, in a framework of political disputes and tension, Uruguay moved from a monopolistic model to a public-private differential system. This article describes the processes, inserts them in the context of the dynamics produced in the region, displays the characteristics, shows the complexity of the tensions and struggles around the construction of university systems and process and conceptualizes a phase of the university dynamics in the country .

Key words : Differentiation. College. Politics. Private universities. Public universities. Reforms.

\section{O GRANDE CICLO DA REFORMA DA DIFERENCIAÇÃO DA EDUCAÇÃO SUPERIOR DO \\ URUGUAI: DA UNIVERSIDADE SISTEMA AO SISTEMA UNIVERSITÁRIO (1984-2013)}

Resumo: A educação superior no Uruguai durante mais de 150 anos estava reduzida a uma única instituição, a Universidade da República, até que em 1984 a primeira universidade privada e a segunda universidade do país foi criada. A partir desse momento começou uma mudança na dinâmica da educação superior, que definimos como a reforma da diferenciação e que envolveu a construção lenta e controversa de um sistema universitário. Foi um período de 29 anos de duração, que terminou em 2013, em que, em um quadro de tensões políticas e lutas, passou-se de um modelo monopólico a um sistema diferenciado público-privado. Este artigo descreve os processos, insere-os no marco 
das dinâmicas produzidas na região, exibe as características, mostra a complexidade das tensões e lutas em torno do processo de construção dos sistemas universitários e conceitua uma fase da dinâmica universitária do país.

Palavras-chave: Diferenciação. Faculdade. Política. Universidades privadas. Universidades públicas. Reformas.

\section{EL CONTEXTO DOMINANTE DE LA EDUCACIÓN UNIVERSITARIA EN URUGUAY}

La educación superior en Uruguay nace en el periodo post colonial en lo que se ha definido como la Universidad de los doctores, caracterizada por ser un modelo de elites, profesionalizante, adscrita al Estado y laico (RAMA, 2006). Se expresó en la creación de la Universidad de la República que inició su proceso fundacional en 1833 con la aprobación de su ley de creación, tuvo un nuevo evento en 1838 cuando se dictó un decreto presidencial por el cual se creó como Universidad Mayor de la República y finalmente se constituyó efectivamente en 1849 por acto de Gobierno cuando se funda como Universidad de la República e inicia sus actividades con su primer curso dictado en 1851. El largo periodo responde a los avatares de los conflictos bélicos y las invasiones externas (ODDONE; PARIS, 1963; BRALICH, 1987; CHIANCONE; MARTÍNEZ, 1997). Formada bajo un modelo napoleónico, la Universidad ofrecía todos los niveles educativos, pero históricamente ha ido perdiendo su rol de servicio público en todos los niveles: en 1877 la Ley de Educación Común se sacó la Enseñanza Primaria de la universidad, en 1935 se separó le Enseñanza Secundaria de la Universidad; en 1984 perdió su monopolio universitario y en el 2012 perdió su monopolio público.

\section{EL MARCO CONSTITUCIONAL DE LA PRESTACIÓN DEL SERVICIO EDUCATIVO}

La Constitución de 1966 aprobó un esquema de libertad en la prestación del servicio educativo en el nivel universitario pero sin embargo los gobiernos democráticos hasta 1973 ni la dictadura hasta 1984 instrumentaron. El artículo 68 de dicha Constitución concebía una lógica de libertad educativa junto al tradicional modelo de Estado docente y en relación al sector privado disponía que "queda garantida la libertad de enseñanza. La ley reglamentará la intervención del Estado al solo objeto de mantener la higiene, la moralidad, la seguridad y 
el orden públicos. Todo padre o tutor tiene derecho a elegir, para la enseñanza de sus hijos pupilos, los maestros o instituciones que desee". En ésta línea de favorecer la expansión de la educación privada, la Constitución en su artículo 69 estableció que las instituciones educativas estarán exoneradas de impuestos, al disponer que "las instituciones de enseñanza privada y las culturales de la misma naturaleza estarán exoneradas de impuestos nacionales y municipales, como subvención por sus servicios. Al tiempo la Constitución impulsaba un modelo público donde eran obligatorias la enseñanza primaria y la enseñanza media, agraria o industrial y donde la ley debía proveer lo necesario para la efectividad de estas disposiciones (Art. 70). Ella al tiempo se incentivaba un modelo de gratuidad en el acceso al declarar de utilidad social la gratuidad de la enseñanza oficial primaria, media, superior, industrial y artística y de la educación física; la creación de becas de perfeccionamiento y especialización cultural, científica y obrera, y el establecimiento de bibliotecas populares (Art. 71) (REPÚBLICA ORIENTAL DEL URUGUAY, 2013). No es estableció en el texto constitucional que esta exoneración estuviera asociada a que fuera gestionado el sector privado por instituciones con o sin fines de lucro, sino que la norma no limitó las formas de su prestación.

\section{LA EDUCACIÓN SUPERIOR DURANTE LA DICTADURA}

En varios países de la región el proceso de diferenciación ha estado asociado a periodos autocráticos o militaristas (El Salvador, Chile, Bolivia). En otros, la diferenciación ha estado asociada a procesos democráticos (Uruguay, Venezuela, Colombia, México o Costa Rica) (RAMA, 2009). En Uruguay la dictadura militar (1973 - 1984) mantuvo el monopolio de la Universidad de la República, prohibió la autonomía y tuvo una dominancia del modelo autoritario sobre el modelo tecnocrático, pero sin modificar la estructura del sistema (BRALICH, 1987). El periodo se caracterizó por la pérdida por renuncia o destitución de 1100 docentes (casi el 40\% de la planta), la clausura o paralización de algunos servicios universitarios y una dinámica de funcionamiento jerarquizada y subordinada al Poder Ejecutivo que impulsó un sistema altamente regulado en reglamentos y normas y con multiplicidad de control (UNIVERSIDAD DE LA REPÚBLICA, 1986). Hubo una caída del presupuesto derivado de la casi eliminación de los docentes de dedicación total y la reducción a una universidad docente a nivel de grado y profesional con casi nula presencia del postgrado.

La presencia de un modelo tecnocrático duró unos pocos años durante los cuales se estableció un sistema selectivo de acceso con un examen de ingreso 
desde 1980 que impactó en la reducción de la matrícula que venía creciendo derivando en foco de resistencia estudiantil y que finalmente fue suprimido en 1983 cuando ya el régimen estaba en fase de terminación. La intervención universitaria mantuvo el esquema básico de 1973 con una sola universidad profesional con un sistema de ingreso abierto y gratuito que terminó conformando una macrouniversidad donde la matrícula pasó de 33.127 en 1973 a 64.086 en 1984 con un incremento anual 6.2\%, centrada en Montevideo y con escasísima presencia regional en un país donde sin embargo cerca del $60 \%$ de la población vive fuera de la capital.

La educación privada bajo éste marco constitucional continuó su expansión en el nivel básico y medio donde ya tenían presencia en el país, pero en el nivel terciario, al no formularse una ley reglamentaria de la norma constitucional o autorizarse instituciones, se mantuvo el modelo monopólico. La restricción a la educación superior privada fue así el eje de la política durante la democracia (1967 - 1973) como durante la dictadura militar (1973-1985).

Las confrontaciones políticas antes del golpe de Estado entre el gobierno y la Universidad de la República, y posteriormente las concepciones centralistas y autoridades de la dictadura militar, determinaron el mantenimiento del modelo monopólico público de la prestación del servicio de educación superior. Sin embargo, en un cambio de política, en 1984, ya en el fin de la larga dictadura el régimen habilitó a la creación de la Universidad Católica, marcando el nacimiento de un nuevo ciclo universitario marcado por la reforma de la diferenciación. Este proceso con sus matices se ha realizado a escala global y en la región (TROW, 1974; BRUNNER, 2000; RAMA, 2009).

\section{CAUSAS Y MODALIDADES DE LOS PROCESOS DE DIFERENCIACIÓN UNIVERSITARIA EN AMÉRICA LATINA}

En general en la región (RAMA, 2006), los procesos de diferenciación universitarias del sector público han estado marcados por dos formas. a) La presión de fuerzas regionales que impusieron la creación de universidades públicas y que conformaron un modelo de regionalización y diferenciación con relativa articulación posterior entre ellos (Bolivia, Ecuador, Argentina, Venezuela, México, Brasil, Chile y Perú). b) Resultado de una selección del ingreso a la institución dominante por motivos políticos derivados de sus acciones políticas o académicos en general derivados de la masificación (Venezuela, Chile, Costa Rica, Perú). Al tiempo que la diferenciación privada ha sido resultado de la presión de fuerzas religiosas y sociales (Paraguay, República Dominicana, Ecuador, 
Bolivia) o de concepciones mismas desde el Estado (Brasil). En cada país se han agregado elementos y combinaciones dadas sus características específicas. En el Uruguay el alto centralismo poblacional y económico de la ciudad capital de Montevideo, el bajo peso relativo de los sectores religiosos e inclusive de la sociedad civil respecto al Estado y la alta asociación de la Universidad pública con un partido político, configuraron el retraso relativo, la lentitud del proceso y las especificidades que ha asumido la reforma de la diferenciación.

\section{EL INICIO DE LA DIFERENCIACIÓN CON LA CREACIÓN DE LA UNIVERSIDAD CATÓLICA DEL URUGUAY}

La fundación de las universidades católicas fueron el inicio de la diferenciación de la educación superior y e inicio de la expansión privada en varios países de América Latina en el siglo XX. En muchos países de la región la primera universidad privada han sido católicas: Argentina (1956), Brasil (1941), Ecuador (1946), Bolivia (1966), República Dominicana (1962), Chile (1988), Colombia (1930), El Salvador (1965), Nicaragua (1960), Panamá (1965), Paraguay (1960), Perú (1917), Venezuela (1953) y Uruguay (1984) (ZAPIOLA, 2005; OROZCO, 2004). Y muchas jesuitas o a ellos confiadas su administración. En todos los países ello marco el fin del monopolio público de una o de varias instituciones y el inicio de un ciclo universitario y de una reforma marcada por la diferenciación, el aumento de la participación privado y el nacimiento de la competencia universitaria. Más allá de múltiples causas internas al mundo religioso que impulsaron esos procesos se puede afirmar claramente que la Universidad privada en América Latina nace gracias a la presión de la Iglesia, y dentro de ella en muchos casos de grupos jesuitas que impulsaron su establecimiento. Esta primera apertura, siempre años después, derivó en la creación de otras universidades privadas laicas y religiosas.

En Uruguay el nacimiento de la Universidad Católica (UCUDAL) fue el resultado de un largo proceso iniciado en el siglo XIX, a través del cual la Iglesia Católica reivindicó permanentemente el derecho constitucional a la libertad de enseñanza a nivel universitario. El antecedente más lejano se remonta a 1876, con la fundación del Liceo de Estudios Universitarios convertido en 1878 por aprobación de gobierno en Universidad Libre y que por problemas económicos y una legislación restrictiva llevo a su clausura posterior. Una nueva iniciativa en 1954 llevo a la fundación del Instituto de Filosofía derivada del Congreso de la Unión Nacional de Educación Católica (UNEC) que había propuesto la creación de un centro católico de estudios superiores. En 1961 el Episcopado uruguayo 
hizo pública una declaración de apoyo a la libertad de enseñanza a todos los niveles y a la erección de una Universidad libre. Desde 1962 el Instituto pasó a depender de la Conferencia Episcopal del Uruguay y finalmente en 1979 fue encomendado a la Compañía de Jesús y se inició el proyecto de creación de la Universidad Católica. En 1981 la Conferencia Episcopal Uruguaya manifestó su voluntad de poner en marcha la creación de una Universidad Católica. En las postrimerías del régimen, el decreto del Poder Ejecutivo 343/984, autorizó su funcionamiento y reconoció sus planes de estudios y programas presentados. Ello fue reafirmado por el Decreto Ley 15.661 del 29 de octubre de 1984 aprobado por el Consejo de Estado que estableció disposiciones para que los títulos profesionales que otorguen las Universidades Privadas, cuyo funcionamiento haya sido autorizado por el Poder Ejecutivo, tengan validez oficial sujetos a determinados estándares básicos y a su aprobación. Con ello se viabilizó la creación de la Universidad Católica del Uruguay “Dámaso Antonio Larrañaga (UCUDAL). Esta con el expreso apoyo papal, obra de la Conferencia Episcopal Uruguaya y confiada a la dirección de la Compañía de Jesús, fue inaugurada el 5 de marzo de 1985 y junto a la reciente democracia, comenzó el inicio de un nuevo ciclo en la política universitaria.

\section{EL LARGO CAMINO DE LA REFORMA DE LA DIFERENCIACIÓN}

\section{a) El periodo $1985-1990$}

Desde el regreso de la democracia el país ha atravesado un largo proceso de diferenciación y de conformación de un sistema universitaria. Se ha considerado que ello ha sido del pasaje de la universidad sistema al sistema de educación superior (CHIANCONE; MARTÍNEZ, 1997). El comienzo de la diferenciación estuvo marcado en líneas generales por un contexto general de restauración universitaria de los poderes y dinámicas existentes antes de la dictadura. Ello fue además la tónica general de la sociedad uruguaya bajo la primera presidencia de Julio María Sanguinetti (1985 - 1990) donde la administración política de esa restauración al tiempo que la construcción de un nuevo futuro. Articular las tensiones entre el pasado y el presente al interior del mundo universitario fueron los ejes de un periodo marcado por la devolución de bienes, recursos, docentes y espacios políticos a una universidad que había estado en el centro de los conflictos en el periodo pre dictatorial, y que al tiempo fue el refugio de miles de repatriados que volvieron al país con el fin de la persecución. La lógica 
era también construir nuevas dinámicas, en tanto las tradicionales relaciones entre la UDELAR y el Estado y los Gobiernos habían sido marcadamente conflictivas en el quinquenio antes del golpe militar (Udelar - Cresal, 1986). La nueva relación llevo a Sanguinetti a ser el primer presidente en décadas recibido en la Universidad estatal. La restauración se expreso en el regreso de las autoridades y el rector anterior del golpe de Estado que asumió el cargo el mismo día que se instaló el Parlamento, sin mucha sustentación legal pero con amplia legitimación política (Sanguinetti, 2012). En este contexto, en marzo de 1985 se promulgó la llamada Ley de Emergencia Educativa (15.739) que restituyó a todos los docentes y administrativos, a la vez dispuso la no lesión de los derechos adquiridos de los demás funcionarios. Fue una complicada lógica de equilibrios entre actores enfrentados que buscaba construir un sistema no suma cero en uno de los centros de la lucha política de los 60. El camino fue la democratización del mundo universitario y en tal sentido se convocó a elecciones universitarias con lo cual se restauró la autonomía y el sistema de poder previo dado por la cogestión, pero modificándose la Ley Orgánica de la UDELAR de 1959 e imponiendo que las elecciones se hicieran definitivamente por voto obligatorio, secreto, bajo el principio de representación proporcional y bajo control de la Corte Electoral que asumió el rol de convocar las elecciones, organizarlas y juzgar (con carácter inapelable) sus resultados. (ACUÑA et al., 2012). La nueva Ley además claramente dispuso la sujeción de la docencia al currículo y los planes de estudio, y prohibió formalmente el proselitismo, sin perjuicio de la libertad de cátedra, promoviendo un camino donde el aula fuera el ámbito de formación exclusivo de competencias y no un espacio de lucha y adoctrinamiento de las ideologías. La ley además estableció también una Comisión Coordinadora de la Educación integrada por la ANEP, el MEC, la UDELAR y las instituciones habilitadas -cuya participación debía ser reglamentada- planteando la necesidad de un sistema coordinado y donde también participarían las instituciones privadas. La necesidad de reconstruir el nivel de recursos financieros de la universidad pública monopólica, impidió pensar en una diferenciación y la creación de una segunda universidad en el interior como históricamente se ha propuesto y que se expresó en un proyecto de ley en el 1985. Así, el presupuesto universitario de la Udelar aumentó 138\% en términos nominales al pasar de 24,8 millones a 59 millones de dólares entre 1985 y 1990, lo cual significó la duplicación de su participación en el presupuesto nacional al subir del $2,5 \%$ al $5 \%$, marcando un camino que ha continuado desde entonces.

La restauración, no fue tampoco de la arquitectura previamente existente, sino que el gobierno mantuvo la diferenciación universitaria iniciada, en pa- 
pel, en 1984, y apoyo la creación de la Universidad Católica del Uruguay que inició ese mismo año de 1985, pero no hubo capacidad política de continuar y consolidar una mayor diferenciación. Ella apenas se expresó en el ámbito público en 1988 en la creación de una nueva institución terciaria pública, el Centro de Diseño Industrial en la órbita del MEC.

\section{b) El periodo 1990 - 1995}

Al inicio del gobierno del Presidente Luis Alberto Lacalle, en 1990, se presentó al Parlamento un Proyecto de Ley de Universidades Privadas, para habilitar la diferenciación institucional más amplia y a la vez establecer un mecanismo de regulación más abierto y ajustado al carácter liberal de la Constitución. El proyecto establecía los requisitos de autorización y funcionamiento de las instituciones privadas, y otorgaba al MEC su control. El proyecto se apoyaba en el artículo constitucional que dispone la libertad de enseñanza sin más limitaciones que "mantener la higiene, la moralidad, la seguridad y el orden público" y ajustado a ello disponía que fuera libre el establecimiento de universidades privadas y que su autorización de funcionamiento fuera otorgada por el Poder Ejecutivo. El anteproyecto de Ley que de hecho regulaba el mandato constitucional creaba la Comisión de Educación Universitaria con dos miembros del Poder Ejecutivo y uno de UDELAR. De hecho se habilitaba una presencia de la universidad sistema sobre el sistema que se pretendía crear.

El proyecto de ley respondía a la existencia de instituciones que solicitaban el uso del término Universidad para calificar sus niveles de enseñanza o instituciones, y a buscar continuar el proceso iniciado con la creación de la UCUDAL, la cual mantenía un espacio monopólico en el sector privado que comenzaba a ser objetado. El Proyecto de Ley quedo engavetado por falta de apoyo de los demás partidos (PC y FA), la resistencia de la UDELAR y la poca disposición de la UCUDAL.

\section{c) El periodo $1995-2000$}

Durante el segundo periodo de Sanguinetti (1995 - 2000) se consolidó la diferenciación de la universitaria a través de la aprobación de un marco institucional de regulación de la educación privada que habilitó la creación de varias instituciones universitarias. Los Decretos 308 y 309 de 1995 fijaron el ordenamiento aún vigente del sistema de enseñanza terciaria fijando los criterios y estándares mínimos de la creación de instituciones y programas. Para ser 
"universidad" se requería la realización de docencia, investigación y extensión en tres áreas disciplinarias mínimas no afines, y para los institutos universitarios dos áreas no afines, aunque las universidades también podían ofrecer estos estudios. La norma definió la características de los programas de grado y de postgrado (sólo especialización, maestría y doctorado), que las $3 / 4$ partes del personal docente debe poseer el grado de la carrera que impartan; que el $10 \%$ del personal académico debe acreditar 5 años de experiencia académica y que la mayoría del personal académico debe ser natural o residente en el país. La oferta se puede realizar previamente y los programas que quieran acceder al reconocimiento deberán someterse al MEC antes del año de su dictado. No se establecieron estímulos y se dispuso que las instituciones fueran asociaciones civiles o fundaciones sin fines de lucro.

La norma creó para asesorar un Consejo Consultivo de Enseñanza Terciaria Privada del MEC, como órgano no vinculante y con representación de Udelar, las privadas, el MEC y la ANEP. Se dio un rol destacado en ese ámbito a las Universidades. Las universidades privadas eran juez y parte, pero la Udelar aunque es miembro, y con opinión preceptiva en relación a la autorización de funcionamiento de las instituciones privadas, no está sujeta a las disposiciones o criterios del órgano, dada la autonomía dada por su marco legal. Se creó entonces una dinámica regulatoria dual no sistémica, lo cual caracteriza en general a la educación superior en la región, y con relativa supervisión de la universidad pública monopólica para entonces.

A partir de ese marco, se autorizaron a varias instituciones existentes con trayectoria docente a acceder al nivel universitario. Así sucesivamente nacieron ORT (1996), UM (1997) y UDE (1998) como universidades y el IAS y el CLAEH como institutos terciarios. Con ello se consolido finalmente un subsistema de educación superior privada e inicio otra fase la educación universitaria en el país. Aunque participó en su formulación, el Decreto fue objeto de un rechazo por la UDELAR ya que ponía en la órbita del Poder Ejecutivo la supervisión del sector terciario privado, que ella pretendía a ella. El recurso fue llevado a la justicia donde fue rechazado. El tema nunca quedo laudado y en el contexto de pugna entre el gobierno nacional y la UdelaR, su delegación no asistió al Consejo por un largo período, y aun luego de reintegrada discrepó con los criterios empleados por los delegados del Poder Ejecutivo en múltiples ocasiones (BENTANCUR, 2004).

También avanzó un casillero, la diferenciación terciaria en el sector público, ya que durante el período se inició un proceso de regionalización de la formación docente terciaria pública mediante la creación de los diversos Centros 
Regionales de Profesores. Antes tal actividad era realizada exclusivamente en Montevideo a través del Instituto de Profesores Artigas. Para entonces, el solamente un tercio de los cargos docentes en el nivel medio estaban ocupados por profesionales titulados del área. Se crearon CERP en las ciudades de Florida, Colonia, Rivera, Salto, Maldonado y Atlántica en Canelones, que cambiaron el panorama de la formación terciaria pública en el interior.

\section{d) El periodo $2000-2005$}

En el gobierno de Batlle continuó la diferenciación institucional y la expansión privada con la creación de 4 institutos universitarios y dos terciarios. La diferenciación se realizó no en el nivel universitario que a partir de entonces se mantuvo en cuatro universidades privadas, sino en la creación de instituciones de nivel terciario, pero por primera vez se habilitaron instituciones terciarias radicadas en el interior. Así, nacieron el Instituto Universitario Francisco de Asís y el Instituto Universitario Maldonado - Punta del Este y el Instituto Politécnico de Valencia (Colonia), que permitieron una regionalización de la educación privada a través de instituciones del interior mismo.

Igualmente en el marco de la diferenciación y de la universitarización de la formación terciaria se impulsó la elevación de los niveles de las instituciones públicas militares, se reconoció el nivel universitario de los egresados de los institutos militares, a través de un mecanismo -que mantenía el monopolio de hecho de una sola Universidad - pero que mediante la realización adicional de tesis, les permitía acceder a nivel de licenciatura universitaria (MARTÍNEZ, 2010). Ello sentó las bases del camino hacia la creación de un subsistema público a través de la Coordinación de Instituciones Terciarias Públicas, impulsadas por el MEC en el 2002.

Dos periodos marcados tuvo el gobierno de Batlle y también dos ministros y políticas universitarias diferenciadas. Una fase pre crisis con un gobierno de coalición y cuyo Ministro del MEC fue Mercader que desarrolló una política de continuidad de iniciativas que venían desde el gobierno de Sanguinetti y Lacalle, y otra fase dada por la crisis y que implicó una detención de las políticas universitarias de diferenciación bajo el Ministro Guzmán. Tal vez se podría decir: una fase de impulso y cambios bajo las ideas liberales de Batlle y otra fase de freno dada por las derivaciones de la crisis y donde se estuvo menos proclive a estrategias diferenciadoras de la Udelar y donde ésta a su vez tuvo un mayor protagonismo en las políticas, al tiempo que el Frente Amplio apoyo las salidas a la crisis. Ello mostró que más allá de los hombres e ideologías, la 
política universitaria está asociada a la gran política nacional y en este caso a la prioridad de construir consensos de superación de la crisis. Hubo entonces un cambio de entonación en la segunda parte del período con una acción gubernamental más asociada a la calidad que a la diferenciación, marcando el fin de la reforma de la diferenciación en el sector público.

\section{e) El periodo de $2005-2010$}

En el 2005 se produce un fuerte cambio político en el país con el triunfo el Frente Amplio que representa a la izquierda uruguaya. El bloque de poder claramente integró a la Universidad de la República que comenzó a tener un fuerte protagonismo en la política universitaria y que fue uno de los soportes centrales de su gobierno. Ello se expresó en la colocación al frente del MEC a diversos cuadros de la UDELAR designando como Ministro de Educación a un ex Rector. En este escenario los mecanismos institucionales de políticas en relación al sector universitario privado pasaran a tener una mayor supeditación a los criterios de la universidad - sistema. El propio presidente del órgano de asesoramiento, el Consejo Técnico Consultivo de la Universidad Privada, fue el representante de la UDELAR, activo militante contra las privadas. A partir de allí se promovió la limitación a la expansión de las privadas al restringir su diferenciación por la vía de limitar y dificultar las autorizaciones de programas, enlenteciendo sus trámites administrativos y poniendo mayores controles administrativos. Ello cerró el ciclo de la diferenciación privada, que pasó, en lo institucional de una ciclo expansión a un ciclo retractivo, más allá de que su matrícula y sus tasas de egresos continuaran expandiéndose.

La orientación de reforzar el rol monopólico de la Udelar y como universidad "mayor" y cabeza del sistema se expresó en múltiples acciones, entre las cuales reducir algunos tibios niveles de diferenciación realizados previamente. Fue así que el Centro de Diseño Industrial (instituto terciario creado por el MEC en 1998) fue traspasado a la Facultad de Arquitectura de la Universidad de la República. También se expresó en un anteproyecto de ley de creación de la agencia de evaluación y acreditación, que no sólo la definía como "arquetipo universitario" sino como ámbito preceptivo de la regulación a las universidades privadas. En este contexto también se redujo la autonomía y los recursos de los Centros Regionales de Formación Docentes de la ANEP, limitándose su regionalización.

El modelo de "universidad - sistema" fue reforzado fundamentalmente por la vía de un mayor nivel de financiamiento que alcanzó dimensiones interna- 
cionales, y que permitió que la UDELAR iniciara con vigor una política de regionalización con el objetivo de constituirse en una universidad nacional. Sin embargo, en este contexto en al ámbito parlamentario proliferaron propuestas de creación de universidades públicas en el interior del país que no fueron apoyadas por el gobierno.

\section{LA DIVERSIDAD DE PROYECTOS PARA IMPULSAR LA DIFERENCIACIÓN PÚBLICA}

La preocupación por la diferenciación del sector público mediante la creación de nuevas universidades en el Uruguay ha sido una constante del sistema político expresada en múltiples proyectos de Ley. Sin embargo, estos han chocado contra las mayorías necesarias en el Parlamento ya que el marco normativo como ente autónomo requiere mayorías especiales. Las iniciativas han provenido fundamentalmente de diputados y senadores que han mirado hacia el interior, tanto del Partido Nacional como del Partido Colorado.

En 1985, Cerchiaro (Partido Colorado) propuso la creación de la Universidad Autónoma del Norte y Litoral Oeste del país. El eje de su propuesta se centraba en la "excesiva población estudiantil que supera ampliamente las posibilidades materiales de la UDELAR así como la centralización de sus institutos en la capital. Argumentaba que tales factores han originado profundos problemas de orden social, como el éxodo de jóvenes que dejen sus departamentos, migración que se hace a costa de inmensos sacrificios familiares como de traumáticas situaciones afectivas.

En el 2006, el Senador Sanguinetti (Partido Colorado) propuso transformar los CERP y la formación docente en un nivel universitario

En 2007, 26 diputados (Partido Nacional) con un enfoque de descentralización y coordinación, propusieron la creación de la Universidad Nacional del Uruguay localizada fuera de la capital de la República con la misma estructura de la Ley Orgánica de la UDELAR y la creación de un Consejo de Coordinación Universitaria presidido por el MEC e integrado con los rectores de las universidades públicas y privadas.

En 2008, el Diputado Amado (Partido Colorado) propuso la creación de la Universidad José Artigas, como ente autónomo y cuyo campus central y los institutos, departamentos, centros y servicios estarían en el interior del país. El proyecto facultaba establecer un sistema por el cual los estudiantes con capacidad contributiva contribuían al financiamiento, establecía un funcionamiento por Departamentos, disponía la evaluación periódica interna y externa, fijaba 
la elección del Rector por voto universal interno pero este podía ser cualquier ciudadano que acreditara capacidad e investigación científica. Y no podría ser reelecto. Establecía un sistema selectivo de acceso en función de estándares mínimos de conocimiento, y promovía una estructura ajustada al Modelo de Bolonia y donde la investigación era obligatoria para los profesores de tiempo completo.

En 2008, el Diputado Delgado (Partido Nacional) propuso la creación de la "Universidad de Formación Docente y Perfeccionamiento Docente" como ente autónomo que sucedía de pleno derecho a la Dirección de Formación y Perfeccionamiento Docente de la ANEP, separando a este ámbito al darle rango universitario a la formación docente. Sostenía que al carecer el país de un ámbito de formación docente en el nivel universitario se desarrollaban los "vicios que han venido afectando a la Universidad: elitismo, pesadez para resolver, politización e inequidad". Era una universidad especializada, con co-gobierno, elecciones secretas, obligatorias y proporcionales, pero "conciliaba con el respeto a los méritos académicos". Se apoyaba en la libertad de cátedra, pero incorporaba en el mismo nivel la laicidad que la concebía su contraparte natural.

En 2010, el Diputado Planchón (Partido Nacional) propuso crear la "Universidad de la República del Interior del País", con 4 regionales en el territorio y como la UDELAR sobre la base del acceso abierto. Al año siguiente, en el 2011, cuatro diputados de los cuatro partidos con representación parlamentaria, propusieron la creación de una "Universidad Autónoma para la Cuenca de la Laguna Merín" en el interior, en la ciudad de Treinta y Tres, y cuyo Consejo Directivo iba a estar integrado por miembros entre otros designados por el Poder Ejecutivo, de los gobiernos departamentales de la cuenca y de los centros comerciales e industriales. El proyecto creaba un Director General designado por concurso de méritos y oposición, los gobiernos departamentales estaban obligados a contribuir al financiamiento y todo el personal seria propuesto por concurso de oposición.

\section{LAS REGIONALIZACIONES FRUSTRADAS DE LA EDUCACIÓN SUPERIOR}

El Poder Ejecutivo en el 2000 autorizó a funcionar como Instituto Universitario a la Asociación Universitaria Maldonado - Punta del Este (IUPE) reconociendo su carrera de Abogacía y en el 2002 las de Notariado y Contador Público. Fue un camino hacia la regionalización de la educación superior en el interior a través de instituciones privadas del propio interior. Esta realidad 
alcanzó al menos a 1.000 estudiantes y derivó en cerca de 500 egresados en las sedes de Maldonado, Durazno, Treinta y Tres, Melo y Rivera, en las cuales dictaba dichas carreras. El ciclo concluyó 10 años después de estar funcionando con reconocimiento expreso del Ministerio de Educación y Cultura (MEC), cuando el Consejo Consultivo de Educación Terciaria Privada (CCETP), con la abstención de los dos representantes de las universidades privadas solicitó al MEC revocar el permiso al IUPE. El presidente del CCETP) expresó que "la institución no debería existir", argumentando que algunos miembros de dicho órgano no habían avalaron el primer permiso que se otorgó en 2000.

La realidad era efectivamente de una institución universitaria que no se manejaba de acuerdo a los criterios académicos requeridos así como de un marco normativo restrictivo y lento de resolución. En dicho contexto el IUPE enfrentó además un juicio por la supuesta venta de un título presentado por el MEC tras una denuncia de un particular y el propio MEC analizó presentar una denuncia penal por considerar que cobró por adelantado cuotas sabiendo que podía ser revocada su autorización para funcionar. Finalmente el Poder Ejecutivo revocó el permiso al IUPE para dictar carreras terciarias, y también firmó un decreto que permitió que sus alumnos pudieran revalidar sus estudios en otras instituciones privadas que tuvieran esas mismas carreras reconocidas. La UdelaR no reconoce los estudios de las privadas y tampoco lo hizo en este caso. Así, los alumnos de Maldonado, la única sede autorizada, pudieron revalidar todas las materias cursadas, y los estudiantes de las demás sedes (Rivera, Durazno y Tacuarembó) pudieron revalidar automáticamente solo hasta dos tercios de la carrera, en tanto que las restantes tuvieran que pasar a ser validadas a través de pruebas en las distintas instituciones privadas. Hubo amplia discusión que se centró en los estudiantes, en el reconocimiento de sus estudios y en la posibilidad que continuaran sus recorridos en las universidades privadas. Se estaba ante un punto en el marco de la inflexión de la reforma de la lenta construcción del sistema universitario. Esta reducción de oportunidades de estudios terciarios en el interior desde el lado privado está en correlación con un amento de la presencia de la universidad pública en el interior en el marco de su lógica de universidad - sistema, en este caso de sistema nacional.

Han existido también otras iniciativas independientes privadas de educación superior en el interior del país a través de instituciones desde el propio interior, tal como la impulsada por la Universidad Politécnica de Valencia de España quien en 1997 promovió la constitución de la Fundación para el Desarrollo del Cono Sur en Colonia en colaboración con la instituciones de España y de Uruguay, que recibió de la Intendencia los terrenos del Hotel Real de San 
Carlos y que desde el 2003 comenzó a ofrecer cursos terciarios propios y en coordinación con las universidades privadas. Con motivo de la crisis de España, desde aquel país se dejó de subsidiar las actividades y en enero del 2012 se interrumpió esta iniciativa del Centro Politécnico del Cono Sur.

Más allá de los problemas de gestión y del apoyo de las diversas intendencias municipales del interior, el fracaso de estas iniciativas muestra la ausencia $-\mathrm{y}$ la necesidad- de sistemas de apoyo nacionales para facilitar estas oportunidades de acceso a la educación superior. Es claro que se necesita una acción decidida de apoyo y de recursos, no solo para que la educación pública tenga presencia en el interior, sino también para que las acciones que llevan adelante individual y solitariamente las universidades privadas desde Montevideo para el interior tengan mayor viabilidad y efectivas esperanzas. Se va con ello, no solo la diversidad, sino también la democracia. Los fines públicos, no solo los realizan los organismos públicos, sino en democracias avanzadas, también las instituciones privadas.

\section{LA REGIONALIZACIÓN DE LA EDUCACIÓN SUPERIOR PRIVADA}

Asociado a la diferenciación a través de universidades privadas, ha habido una creciente presencia de ellas en el interior del país. Así, la ORT mantiene un convenio con el Instituto Tecnológico y desde 1994 apoya el dictado de carreras cortas, suministra planes y materiales y corrige exámenes y certifica en Colonia, Maldonado, Paysandú y Salto. El CLAEH instaló un centro en Maldonado desde donde desde el 2008 inició la primera Carrera de Medicina privada en el país y en el interior; la UDE desarrolla cursos técnicos desde el 2007 de su Facultad de Ciencias Agrarias y en el 2013 se instala en Colonia ofreciendo dos carreras de grado (Administración y Contaduría). La UCUDAL lleva adelante una amplia oferta de carreras de grado y técnicas a través de sus sedes de Salto y Punta del Este, y tiene además una presencia activa en Florida, Paysandú y Trinidad. Además en el 2009 se constituyó el Cluster Punta del Este: Ciudad Universitaria que propende a crear sinergias para hacer del balneario un centro académico y en el cual participan todas las instituciones allí instaladas junto a la Intendencia Departamental de Maldonado, la Liga de Punta del Este, la Cámara Empresarial de Maldonado y Santander Universidades.

La reforma de la diferenciación institucional se expresa también en una regionalización y con ello cubrir las demandas de acceso y resolver la amplia inequidad de acceso regional del país. En Uruguay este ha sido un proceso 
lento y atrasado, y esta regionalización privada casi sólo ha sido posible en muchos casos gracias al apoyo de las Intendencias municipales del interior que han facilitado becas, apoyos o aulas. Las 18 intendencias del interior han tenido históricamente políticas de educación superior con distinta intensidad: antes -y aún- a través de residencias estudiantiles en Montevideo, y ahora apoyando a las universidades para ofertar o instalarse en sus departamentos. Al tiempo éstas están lentamente cambiando su perfil y su trabajo para conformar dinámicas organizativas, cuadros docentes, enfoques académicos y redes de cooperación cada vez más nacionales y con más participación del interior. Es una demanda y una necesidad derivada también de una nueva dinámica económica impulsada desde el campo con crecientes aportes de valor agregado de capital humano y de conocimiento.

La matriz universitaria tradicional en el país es centralista y Montevideana, asociado a un modelo económico proteccionista y estatista, y de un paradigma tradicional de la calidad de tipo presencial. En el interior, las bajas escalas o la falta de docentes, requeriría marcos que permitieses ofertar cursos intensivos, educación semipresencial, cursos virtuales, así como uso de locales diversos como hoteles u otros. Algunas intendencias han iniciado el camino y han construido aulas para que todas las instituciones puedan ofertar sus servicios educativos. La dinámica actual de repetir las infraestructuras por parte de cada una de las instituciones o no habilitar la flexibilidad de ofertas virtuales, limita la diferenciación como regionalización y construir efectivos sistemas universitarios nacionales.

\section{CAMINOS Y DEBATES DE LA REFORMA UNIVERSITARIA}

El regreso democrático en 1985, planteo a la Universidad de la República, más allá de la restauración de autoridades, capacidades y autonomías, la necesidad de pensar su futuro. El primer diagnóstico universitario realizado a la salida de la dictadura (Udelar, 1986) mostraba en lo académico la carencia de núcleos académicos; la rigidez institucional y burocrática de funcionamiento; la masificación de la matrícula sin contrapartida docente y locativa; la insuficiencia de recursos económicos estatales y de fuentes complementarias y el bajo contacto con las comunidades científicas internacionales. Y también veía inexistencia de definiciones convergentes sobre la necesidad de cambios y escasa percepción universitaria de los riesgos asociados al sostenimiento de la situación institucional. Visualizaba un escenario nacional favorable con la posibilidad de múltiples apoyos desde la sociedad, un marco político menos 
polarizado y con más capacidad de dar respuestas a las demandas y un auspicioso nuevo contexto económico y social.

Diagnosticaba claramente la herencia dictatorial y también la ventana de oportunidades que se abría en la restauración democrática, a partir de un análisis prospectivo y de reflexión sobre los futuros posibles. La pérdida del monopolio universitaria en 1984 con la habilitación de la Universidad Católica del Uruguay planteaba un cambio y una ruptura en el escenario. Se iniciaba una reforma universitaria dada por la diferenciación que iba a recorrer un largo tiempo histórico entre 1984 y el 2013 donde se paso de una dinámica monopólica de una universidad - sistema a un sistema universitario diferenciado con pluralidad de instituciones con sus propios roles y características.

El futuro desde la universidad se pensó bajo un escenario prospectivo de cuatro escenarios posibles: una universidad profesionalizante - modernizada, que mejoraba la formación docente pero que se mantenía como una institución no generadora de conocimiento; una universidad bloqueada, que no alcanzaba consensos estratégicos internos, se encerraba en conflictos e iniciaba un camino de fuerte burocratización que dificultaba aún más la toma de decisiones; una universidad sustituida, derivada de la irrupción en el ámbito privado y público de nuevas instituciones derivadas de la lentitud o incapacidad de la institución de asumir las reformas necesarias, y una universidad integrada tanto internamente entre los diversos grupos de poder y académicos que permitía una síntesis y una acción dinámica, como también externamente a partir de una dinámica articulación colaborativa con los diversos estamentos de la sociedad.

Ese debate sin embargo quedo acotado primero en la compleja restauración que marcó el periodo de Lichtenstejn (1985 - 1989) aunque tuvo una huella en 1988 en las Jornadas de Solís convocadas por el rectorado. Posteriormente quedó congelado ante la renuncia del Rector y la ascensión al cargo de Brovetto quién marcó el inicio de una hegemonía burocratizada y conservadora al interior de la Udelar e impuso la dominancia de los dos primeros escenarios prospectivos referidos.

En 1993, probablemente hartos ya de estar hartos, luego de cuatro años desde 1989 de múltiples intentos frustrados en el seno de la Udelar de generar una discusión e impulsar una amplia reforma universitaria, cuatro decanos (Wschebor, Touyá, Diaz y Scarsi) hicieron pública una larga carta al Rector. La política de Brovetto de evitar una discusión interna llevó a quienes procuraban construir una corriente de transformación universitaria, a formular un documento público con miras a potenciar la discusión y poner en la mesa las reformas necesarias. Era una pelea por espacios de poder e ideas, entre conservadores 
y reformistas, entre poder central y descentralización, entre reformas y status quo. Entre quienes sostenían que se había agotado el impulso renovador que llevó a la promulgación de la Ley de la Universidad de 1958 y donde varios de ellos mismos habían participado. Formulaban un espíritu renovador y cambios en la política interna, pero también reconocían, intrincadamente, la necesidad de fundar una nueva institucionalidad. Este espíritu fracaso y las fuerzas conservadoras impusieron su proyecto de rechazo a cualquier diferenciación institucional.

\section{EL FIN DEL LARGO CICLO DE LA DIFERENCIACIÓN UNIVERSITARIA}

En el periodo iniciado en el 2010 bajo la presidencia de Mujica, finalmente se produjo la diferenciación pública a partir de la aprobación en noviembre de 2012 de la Ley N 19.043 que creó la Universidad Tecnológica del Uruguay (UTEC) radicada en el interior y la segunda universidad del país. Aprobado por 79 votos en 93 (se necesitaban 66), el proyecto presentado por el gobiernos de la UTEC, es el tercer ente autónomo de la enseñanza luego de la Universidad y el Consejo Directivo Central (primaria, media y técnica). Su creación marca un hito en el largo ciclo de la reforma de la diferenciación universitaria y pone el cimiento al fin de este ciclo iniciado en 1984 cuando se rompió el monopolio de una sola institución. La creación fue también un momento de definiciones y de apertura a nuevos modelos educativos, y que más allá de sus limitaciones, marcará los nuevos escenarios futuros que sin duda pasarán a centrarse en los problemas de la calidad, la pertinencia y la eficiencia en el gasto.

La discusión legislativa fue una radiografía de las diferencias de modelos de cómo proyectar el país al futuro. A último momento el Partido Nacional cambió su posición negativa inicial, y permitió al Frente Amplio alcanzar los votos requeridos. Dos diputados del Partido Independiente y un solitario voto colorado acompañaron el proyecto. El Partido Nacional argumentó que aún a pesar de discrepar con el modelo de dirección cogobernada, la creación de una segunda universidad en el interior, era un reclamo largamente expresado, y que por ello no podría obstruir su creación. Tal posición a su vez expresó, las características de la diferenciación universitaria pública y la discusión al respecto a las características de la diferenciación o del sistema universitario. Las posiciones eran varias: mantener el monopolio de la Udelar y que esta continuara el proceso de cubrir demandas del interior, crear una universidad autónoma, idéntica a Udelar con una presencia institucional de esta en la dirección, una universidad idéntica pero en el interior y especializada, o una 
universidad que incorpore diferenciaciones respecto al llamo modelo "arquetipo de la Udelar" con algunas diferenciaciones, vinculadas a un modelo de gestión menos corporativo interno y con mayor presencia de la sociedad civil y de la sociedad política. Finalmente el proyecto aprobado, se orientó a la última de las características, pero sin la presencia, ni aún formal, de la sociedad política.

La ley aprobada establece que la UTEC es autónoma, cogestionada con autoridades elegidas por docentes, estudiantes y egresados y con un consejo directivo compuesto por el rector, dos docentes, dos estudiantes, un representante de los trabajadores y otro de los empresarios, y los directores de los Institutos Tecnológicos Regionales (ITR). Estos, como unidades académicas especializadas en un área del conocimiento vinculada con el perfil productivo de la zona en la que se instalen. Se mantuvo un modelo universitario autónomo y colegiado de tipo corporativo pero agregando otras representaciones, en este caso externas, como trabajadores y empresarios. Mostró una diferenciación no solo institucional, sino relativamente de la tipología organizacional. Sin duda a futuro, la UTEC con los años tendrá su propia regionalización y las fuerzas políticas y sociales le impulsarán a tener muchos centros regionales, tal vez hasta 18, haciendo la presencia corporativa externa menor, un mayor peso en las pertinencias locales, un mayor poder de los espacios regionales y probablemente una mayor articulación a las Intendencias del Interior.

Finalmente, en el 2013, el ciclo se terminará con la creación de la Universidad de Educación (UNED), actualmente en discusión parlamentaria pero con decisión política de aprobación como un ente autónomo. Ella implicará el desmembramiento de los institutos terciarios de formación terciaria del CODICEN, y su transformación como universidad. Probablemente se siga el camino de una diferenciación limitada iniciada por la UTEC con sus propias especificidades. En este caso, es también la conclusión de un largo camino marcado por muchos hitos, entre los cuales, los CERP, varias iniciativas de proyectos de ley, los artículos 31, 49 y 107 de la Ley General de Educación $\mathrm{N}^{\mathrm{o}}$ 18.437 (2008) así como un verdadero clamor de los docentes. El fin del ciclo de la reforma de la diferenciación: el legado de Mujica

\section{CONCLUSIONES}

Uruguay inició como en toda la región el proceso de diferenciación en forma muy tardía posterior a la dictadura y sujeto a variables más políticas que educativas. El pasaje de la universidad sistema al sistema universitario, fue el eje central de la política universitaria durante casi tres décadas, marcado por un 
continuo proceso de avances con constantes resistencias políticas. La diferenciación al tiempo estuvo articulada y en tensión alrededor de la problemática de la centralización y la baja regionalización de los accesos a la educación superior.

Definimos como diferenciación un proceso por el cual se pasó de una universidad que cumple todas las funciones universitarias del país, a la existencia de al menos tres universidades públicas y privadas, que permiten dinámicas sistémicas complejas y lógicas de competencia institucional con diversidad de segmentaciones. La existencia de al menos 6 instituciones y dos sectores (público y privado) permite asumir que se está ante un sistema diferenciado. Sin duda, contemporáneamente la dinámica tiende a incluir instituciones de educación a distancia y universidades internacionales.

La reforma se caracterizó por una fase inicial de de diferenciación privada (1984 - 2009) en la cual se crearon cuatro universidades y posteriormente una fase de diferenciación pública (2009 - 2013) en el cual se han creado dos universidades. La diferenciación se tornó una variable política y por lo tanto sujeta a las dinámicas políticas de apoyo o rechazo, con un actor preponderante como es la Udelar. Esta diferenciación al mismo tiempo introdujo en su primera fase el debate y las tensiones sobre la regulación y el rol de la universidad pública monopólica sobre los demás actores, en tanto posteriormente introducirá la tensión alrededor de la distribución de los recursos públicos.

La diferenciación se ha desarrollado por la vía de un proceso lento de apertura diferenciado a instituciones terciarias y universitarias. También la diferenciación o la apertura a otros proveedores se ha dado en forma más amplia a los niveles de ofertas de grado, y muy escasamente a los postgrados, y entre ellos fundamentalmente al nivel de doctorado.

La diferenciación no ha sido meramente institucional, y el debate y las tensiones han girado alrededor del grado de homogenidad y diversidad del sector público. La dinámica muestra, como en toda la región, que la diferenciación pública tiende a introducir nuevas formas de participación externa en las tradicionales configuraciones autonómicas, más allá de que en el Uruguay fueron leves.

La terminación de la reforma de la diferenciación y la conformación de un nuevo marco institucional con un sistema universitario en funcionamiento consolida un escenario más competitivo futuros tanto en el sector público como privado, así como entre ambos. La competencia por recursos, por el valor de las certificaciones, las distintas posiciones de mercado, de ofertas o de niveles de calidad, marcaran los escenarios futuros e impulsarán una nueva fase más centrada en la calidad resultado de un panorama diferenciado en lo institucional. 


\section{REFERENCIAS}

ACUÑA, Javier et al. La educación superior en Uruguay. En: Red Iberoamericana de Investigaciones en políticas públicas, 2012. La educación superior en el MERCOSUR: Argentina, Brasil, Paraguay y Uruguay hoy. Buenos Aires: Biblios, 2012.

BENTANCUR, Nicolás. Cinco dilemas universitarios de comienzos de siglo. Revista Uruguaya de Ciencia Política, Montevideo, n. 14, 2004.

BRALICH, Jorge. Breve historia de la educación en el Uruguay. Montevideo: CIEP, 1987.

BRUNNER, José Joaquín. Educación Superior: desafíos y tareas. Paris: UNESCO, 2000.

BUCHELI, Marisa. EI empleo de trabajadores con estudios universitarios y su prima salarial. Montevideo: UDELAR. Departamento de Economía, 08/00, 2000.

CHIANCONE, Castro Adriana; MARTÍNEZ, Larrechea Enrique. El tránsito de la universidad al sistema de educación superior en Uruguay: aportes al estudio comparado de las políticas públicas de acreditación de instituciones. Revista Educación Superior y Sociedad, Caracas, v. 8, n. 1, p. 23-40, 1997.

MARTÍNEZ, Enrique L. La educación superior de Uruguay en transición. Inercias y horizontes de cambio. Montevideo: Fundación de Cultura Universitaria, 2010.

MARTÍNEZ, Enrique L.; CHIANCONE, Castro Adriana. La educación Superior en Uruguay: matriz inercial y escenarios de cambios". Revista de Innovación Educativa, México, v. 11, n. 57, 2011.

OROZCO, Luis Enrique. Las universidades religiosas en América Latina. Caracas IESALC, 2004.

ODDONE, Juan; PARIS, Blanca. Historia de la Universidad de Montevideo. La universidad viaje 1849 - 1885. Montevideo: Udelar, 1963.

RAMA, Claudio. La tercera reforma de la educación superior en América Latina. Buenos Aires: FCE, 2006. 
RAMA, Claudio. La universidad latinoamericana en la encrucijada de sus tendencias. UNICARIBE, Santo Domingo, 2009.

REPÚBLICA ORIENTAL DEL URUGUAY. Constitución de la República de 1967 (con las modificaciones de 1989, 1994, 1996 y 2004). Disponible en: $<$ http://200.40.229.134/constituciones/const004.htm>. Acceso en: 17 jun. 2013.

TROW, Martín. Problems in the transition from elite to mass higher education [Los problemas de la transición de la enseñanza superior de elite a la de masas]. En: ORGANIZACIÓN DE COOPERACIÓN Y DESARROLLO ECONÓMICOS (Comp.). Policies for higher education. París: OCDE, 1974. p. 51-101.

UNIVERSIDAD DE LA REPÚBLICA. Dirección general de Planeamiento Universitario. La educación superior en Uruguay. Caracas: CRESALC-UNESCO, 1986.

ZAPIOLA. Las universidades católicas en América Latina. Caracas: IESALC, 2005. 